\title{
New knowledge on the impact of sleep disturbances illustrates the urgent need to address sleep problems in nursing home residents
}

It is well-established that sleep disturbances are common among people with dementia living in nursing homes (NHs) (Forbes et al., 2014; Kinnunen et al., 2017; McCleery et al., 2016; Webster et al., 2020). Measuring sleep disturbances or other conditions without pathognomonic manifestations in this population is difficult due to the patients' reduced capacity to give valid self-report. For instance, a person with dementia may not remember that she or he had disturbed sleep and by extension it is not clear whether the patient perceives it as a problem.

Due to communication and assessment issues, most studies investigating sleep in people with dementia use proxy raters or actigraphy to measure sleep. The proxy rater is often the patient's primary nurse who knows the patient well and can answer on the patient's behalf (Husebo et al., 2016). An actigraph is a watch-like device that measures activity/ inactivity (wake/sleep) and is scored using an algorithm-based software (Blytt et al., 2017). To illustrate the discrepancy that may arise between measurements, a recent meta-analysis of 55 studies with a total of 22780 participants showed that $20 \%$ of NH residents with dementia had clinically significant sleep disturbances when assessed with validated questionnaires. However, the same study reported a prevalence of $70 \%$ when sleep disturbances was measured using actigraphy (Webster et al., 2020). When using actigraphy, activity is recorded over 24 hours, providing an objective estimate of sleep. Although the actigraphy have methodological issues, it is a clear advantage that it provides information on periods when the $\mathrm{NH}$ staff is not observing the patient (Gibson et al., 2019).

Although several studies have examined different treatment strategies for sleep disturbances in people with dementia (Blytt et al., 2018a; 2018b; Forbes et al., 2014; Hjetland et al., 2020; Kinnunen et al., 2017; McCleery et al., 2016), few studies have investigated the impact of these sleep disturbances on the physical and psychological well-being of people with dementia. In the article "The impact of sleep disturbances on care home residents with dementia: the SIESTA qualitative study," Webster et al. (2020) ${ }^{1}$ interviewed 18 nurses and care assistants of about 54 patients with dementia and sleep disturbances. By doing so, Webster et al. provide new and important knowledge about sleep disturbances in people with dementia.

The authors recruited four care homes in the Greater London area and included staff providing direct care to the patients; nurses and care assistants who currently or previously worked a combination of day and night shifts at the care home. The staff functioned as proxy raters for the patients and were asked to concentrate on three residents with dementia and sleep problems who they knew well enough to speak about in-depth. The staff was asked questions regarding the sleep of these residents, perceptions of the underlying reasons for the poor sleep, how the poor sleep affected the residents and staff, whether the disturbances persisted, and how sleep disturbances were managed at the NHs. The authors audio recorded and transcribed all of these semi-structured interviews, which was then analyzed.

Through the analysis, the authors identified the following five themes of the impact of sleep disturbances: (1) descriptions and interpretations of sleep disturbances, (2) impact on residents with sleep disturbances, (3) impact on other residents, (4) impact on staff, and (5) impact on relatives. The study includes several descriptions and interpretations of sleep disturbances. These reveal that some residents did not get out of bed during the night but were still identified as awake by $\mathrm{NH}$ staff because they made noise or were visibly awake during hourly observations. Furthermore, there were descriptions of patients getting up during the night, walking and moving around.

The authors interviewed participants about patients who have dementia and sleep disturbances. It is, however, important to highlight that inviting the staff to select cases may lead them to discuss the most vivid examples of sleep problems in their unit. Many patients may be lying still in bed during the hourly observation rounds, and this may be viewed

${ }^{1}$ Henceforth referred to as "the authors". 
as sleep by the observer, even though they are awake. Thus, they may be experiencing sleep difficulties but not being able to communicate this to the NH staff. Previous studies have investigated different assessment methods and reported significant discrepancies between sleep disturbances identified by NH staff using validated proxy rater assessment tools and actigraphy recordings (Blytt et al., 2017). Identifying sleep problems in people with dementia remains elusive - systematic observation is important, and actigraphy may also serve a purpose. However, regardless of the method, those with reduced mobility and absence of vocalization may be overlooked. In light of this, it would have strengthened the study if the authors had included different systematic sleep assessment methods prior to their interviews, such as actigraphy recordings of sleep over a period of 7 days, in addition to systematic observation. It would be of interest to see whom the staff identified as having a sleep problem; was it primarily those with high levels of agitation, making the wakefulness plainly obvious? This would have allowed for an interesting and valuable comparison regarding the different assessment methods as well as insights to what symptoms the staff may overlook.

Regarding the impact on residents from disturbed sleep during nighttime, several issues are addressed. As reported by the authors, the $\mathrm{NH}$ staff describes that patients may display agitation and aggressiveness. The $\mathrm{NH}$ staff also reported that residents were more physically aggressive the day after poor sleep and staff also linked the poor sleep to patients' agitated mood. Reduced mood and irritability are well-known consequences after one or more nights with poor sleep, and it is thus natural to assume that these accounts are valid. However, it should be noted that NH staff compared patients referred to as "poor sleepers" to patients with dementia who they identified as "good sleepers". The following are the two concerns regarding this: First, as pointed out by the authors, the direction of causality is unknown, and second, previous research indicates that $\mathrm{NH}$ staff are not able to identify sleep disturbances as well as actigraphy (Blytt et al., 2017). Therefore, the reported findings on the impact of sleep disturbances should be interpreted with some reservation. This is especially important since the symptoms during daytime could be related to other factors such as pain, hunger, or anxiety. This implies that in some cases the NH staff might assume that the sleep difficulty resulted in agitation and aggressiveness. However, these symptoms might have emerged from other reasons. In light of this, assessment is fundamental. The $\mathrm{NH}$ staff should use validated assessment tools to further investigate the symptoms, including possible secondary causes.
Every person who has been at an $\mathrm{NH}$ has probably observed patients sitting in a chair while sleeping. This image has unfortunately become a cliché in the depiction of $\mathrm{NH}$ patients, illustrating how pervasive daytime sleepiness is. This is also described in the authors' study, as sleeping during the day was listed as one of the unfortunate outcomes of poor sleep during nighttime. As pointed out in the article, this may have negative consequences for patients, since they may become too sleepy to eat and drink properly. This may in turn have manifold consequences depending on the patient's health. For instance, some patients may become dehydrated, some might lose weight, or the reduced food intake may have implications for the management of medical health conditions such as diabetes. Furthermore, sleep during daytime might make it difficult to sleep well during the night, which again may lead to a downward spiral regarding sleep and health among people with dementia living in NHs.

Interestingly, the "poor sleepers" also affected the sleep of other residents. The NH staff reported that patients were sometimes screaming, shouting, or entering other patients' rooms, which naturally impacts the other patients' sleep. This finding highlights the importance of treating sleep disturbances in this patient group, since patients' poor sleep may have spillover effects on other patients, thus making it a broader problem. The impact of this problem is further highlighted when the $\mathrm{NH}$ staff describes the impact that patients' sleep disturbances had on the $\mathrm{NH}$ staff. Not surprisingly, the NH staff described it as particularly difficult to care for patients with sleep disturbances when they disturbed or woke up other patients. As proposed in the authors' study, this often leads to a feeling of being stressed and guilty.

The great impact that sleep problems were described to have on the patient, their NH neighbors, and staff shows how pervasive and far-reaching this issue may be. At the same time, it also illustrates how impactful proper management of these symptoms can be. By developing good and effective treatments for sleep disturbances in this patient group, a broader range of positive outcomes may follow, also for $\mathrm{NH}$ staff. Considering these potential positive outcomes, treating sleep disturbances among $\mathrm{NH}$ patients with dementia should be a clinical priority.

The study by Webster et al. (2020) increases our knowledge on sleep among people with dementia. Focusing on the real-life consequences, when treating sleep, should be a part of the future treatment studies, as it may uncover changes that proxy-rated instruments may not fully capture. It may also be of interest to focus on the experiences of people with dementia who are still capable of providing some self-report. 


\author{
KJersti Marie BlytT ${ }^{1,2}$ AND \\ Elisabeth Flo-Groeneboom ${ }^{3}$ \\ ${ }^{1}$ Department of Health and Caring Sciences, Western \\ Norway University of Applied Sciences, Bergen, Norway \\ ${ }^{2}$ Department of Global Public Health and Primary Care, \\ University of Bergen, Bergen, Norway \\ ${ }^{3}$ Department of Clinical Psychology, University of \\ Bergen, Bergen, Norway
}

\section{References}

Blytt, K. M., Bjorvatn, B., Husebo, B. and Flo, E. (2017). Clinically significant discrepancies between sleep problems assessed by standard clinical tools and actigraphy. BMC Geriatrics, 17(1), 253.

Blytt, K. M., Bjorvatn, B., Husebo, B. and Flo, E. (2018a). Effects of pain treatment on sleep in nursing home patients with dementia and depression: a multicenter placebo-controlled randomized clinical trial. International Fournal of Geriatric Psychiatry, 33(4), 663-670.

Blytt, K. M., Husebo, B., Flo, E. and Bjorvatn, B. (2018b). Long-term pain treatment did not improve sleep in nursing home patients with comorbid dementia and depression: a 13-week randomized placebo-controlled trial. Frontiers in Psychology, 9, 134.

Forbes, D., Blake, C. M., Thiessen, E. J., Peacock, S. and Hawranik, P. (2014). Light therapy for improving cognition, activities of daily living, sleep, challenging behaviour, and psychiatric disturbances in dementia. Cochrane Database of Systematic Reviews, Issue 2. Art. No.: CD003946. doi: 10.1002/14651858.CD003946.pub4

Gibson, R. H. and Gander, P. H. (2019). Monitoring the sleep patterns of people with dementia and their family carers in the community. Australasian fournal on Ageing, 38(1), 47-51. doi: 10.1111/ajag.12605

Hjetland, G. J., Pallesen, S., Thun, E., Kolberg, E., Nordhus, I. H. and Flo, E. (2020). Light interventions and sleep, circadian, behavioral, and psychological disturbances in dementia: a systematic review of methods and outcomes. Sleep Medicine Reviews, 52, 101310.

Husebo, B. S., Achterberg, W. and Flo, E. (2016). Identifying and managing pain in people with Alzheimer's disease and other types of dementia: a systematic review. CNS Drugs, 30(6), 481-497.

Kinnunen, K. M., Vikhanova, A, and Livingston, G. (2017). The management of sleep disorders in dementia: an update. Current Opinion in Psychiatry, 30(6), 491-497.

McCleery, J., Cohen, D. A. and Sharpley, A. L. (2016). Pharmacotherapies for sleep disturbances in dementia. Cochrane Database of Systematic Reviews, Issue 11. Art. No.: CD009178. doi: 10.1002/14651858.CD009178.pub3

Webster, L. et al. (2020). Measuring the prevalence of sleep disturbances in people with dementia living in care homes: a systematic review and meta-analysis. Sleep, 43(4), zsz251. doi: 10.1093/sleep/zsz251

Webster, L., Powell, K., Costafreda, S. G. and Livingston, G. (2020). The impact of sleep disturbances on care home residents withdementia: the SIESTA qualitative study. International Psychogeriatrics, 32, 839-847. 